\title{
ANÁLISE PROBABILÍSTICA DO TRANSPORTE UNIDIMENSIONAL DE CONTAMINANTES EM MEIO POROSO SATURADO USANDO SIMULAÇÃO DE MONTE CARLO
}

Vanessa Almeida de Godoy ${ }^{1}$

Gian Franco Napa García ${ }^{2}$

Resumo: Apesar de as águas subterrâneas possuírem grande importância estratégica, vêm sofrendo várias pressões em decorrência do aumento da quantidade de áreas contaminadas por atividades industriais, urbanas e agrícolas. Com isso, é urgente um gerenciamento que leve em consideração tanto a probabilidade de ser identificada uma concentração superior à uma concentração aceitável, $\mathrm{P}_{\mathrm{f}}$, quanto os custos da contaminação, i.e. que considere o risco relacionado com as áreas contaminadas. No entanto a determinação da $P_{f}$ é complexa devido às incertezas intrínsecas dos parâmetros associados ao transporte de contaminantes. Assim, neste artigo busca-se mostrar um exemplo de aplicação da obtenção da $P_{\mathrm{f}}$ através de uma análise probabilística do transporte unidimensional de contaminantes em meio poroso saturado, usando simulação de Monte Carlo. Os resultados mostram que pequenas variações na concentração inicial (de 10 para $15 \mathrm{~mm} / \mathrm{m}^{3}$ ) podem levar a $P_{f}$ a um incremento de até quatro ordens de grandeza. Além disso, com o aumento do tempo de análise ocorre aumento das $P_{f}$ para uma mesma concentração inicial. Este trabalho evidencia a necessidade de rápida contenção e identificação de vazamentos, reduzindo as $P_{f}$ e consequentemente, os riscos envolvidos. Por fim, espera-se estimular o uso de análises probabilísticas permitindo um melhor gerenciamento das águas subterrâneas e, consequentemente, de toda a bacia hidrográfica.

\footnotetext{
${ }^{1}$ Mestranda do Programa de Geotecnia da Escola de Engenharia de São Carlos da Universidade de São Paulo (valmeida@usp.br). ${ }^{2}$ Doutorando do Programa de Geotecnia da Escola de Engenharia de São Carlos da Universidade de São Paulo (gnapa@sc.usp.br).
} 
Palavras-Chave: Análise Probabilística. Transporte de Contaminantes. Simulação de Monte Carlo.

\section{INTRODUÇÃO}

As águas subterrâneas possuem grande importância estratégica, principalmente em países que sofrem com escassez hídrica e ambiental. No entanto, esse recurso vem sofrendo várias pressões em decorrência do aumento da quantidade de áreas contaminadas por atividades industriais, urbanas e agrícolas. Com isso o gerenciamento dessas áreas tem se tornado essencial na manutenção da qualidade dos recursos hídricos subterrâneos (Hirata, 1993). No entanto, a gestão de áreas contaminadas não é apenas uma questão de comprovação se um local está ou não contaminado e se um local deve ser remediado, mas também como e se o site deve ser corrigido e quais os custos dessa contaminação (LEMMING, FRIIS-HANSEN e BJERG, 2010). Envolvendo, então, a análise, avaliação e gerenciamento de risco. O risco é definido como a probabilidade de falha i.e. probabilidade de ser identificada uma concentração superior à uma concentração objetivo, ou aceitável, determinada por algum órgão ambiental, multiplicada pelos custos esperados de falha (consequências), dessa contaminação (FREEZE et al., 1990 e ISSMGE, 2004). Os resultados de uma boa análise de risco subsidiarão as tomadas de decisões e permitirão a redução dos impactos sobre as águas subterrâneas e, consequentemente, sobre toda a bacia hidrográfica.

Nota-se que a determinação da probabilidade de determinado contaminante estar acima dos valores aceitáveis para água subterrânea é uma etapa essencial na análise de risco. No entanto, para a determinação da probabilidade de falha é necessário que se conheça os mecanismos de transporte de contaminantes e de fluxo de água na área, o que nem sempre se dá de maneira simples. Isto porque os sistemas físicos possuem incertezas intrínsecas (ou inerentes) associadas, i.e., incertezas correspondentes à 
aleatoriedade natural dos fenômenos físicos, químicos, biológicos e atmosféricos aos quais possam estar relacionados (HAMED, BEDIENT e CONTE,1996). A incerteza dos parâmetros físicos em problemas de transporte de contaminantes em subsuperfície é ubíqua. Ela se manifesta desde a heterogeneidade de base da formação do aquífero até a incerteza relativa às propriedades químicas, físicas e biológicas do contaminante a ser libertado e transportado. Além disso, estes parâmetros podem variar muito de um local para outro e também apresentam grande variabilidade espacial no mesmo site (HAMED, BEDIENT e CONTE,1996; LI et al., 2005, BAALOUSHA e KÖNGETER, 2006).

Mesmo com significativa incerteza relacionada ao transporte de contaminantes, os modelos determinísticos foram, por muitos anos, os mais usados para previsão do transporte de contaminantes. No entanto, esses modelos são incapazes de levar em conta as incertezas intrínsecas do meio (HAMED, BEDIENT e CONTE,1996). Com isso houve um significativo avanço na área de modelagem probabilística e estocástica do fluxo de água subterrânea e transporte de contaminantes nas últimas décadas (SITAR e CAWLFIELD, 1987; JANG, SITAR e KIUREGHIAN, 1994; HAMED, BEDIENT e CONTE,1996; Ll et al, 2005, BAALOUSHA e KÖNGETER, 2006). As implicações dessas incertezas intrínsecas são particularmente importantes na avaliação das várias possibilidades de gerenciar o problema. Desta forma, por mais que seja necessário um esforço significativo para incorporar incertezas no processo de análise da contaminação, isso pode resultar no fornecimento de informações úteis e confiáveis na tomada de decisões. Assim, neste artigo busca-se mostrar um exemplo de aplicação da obtenção da probabilidade de falha por meio de uma análise probabilística do transporte unidimensional de contaminantes em meio poroso saturado por meio da simulação de Monte Carlo. Com isso espera-se estimular o uso de análises probabilísticas que são capazes de incorporar as incertezas intrínsecas relacionadas ao transporte de contaminantes e permitem um melhor gerenciamento do problema. 


\section{TRANSPORTE DE CONTAMINANTES EM MEIOS POROSOS SATURADOS}

A interação solo-soluto é de grande complexidade, pois podem ocorrer simultaneamente fenômenos físicos, químicos e biológicos. Essa complexidade, somada ao aspecto transiente dos fluidos e à grande variabilidade das condições geológicas e hidrogeológicas, fazem com que a formulação matemática do movimento de contaminantes seja bastante intrincada. Para lidar com essa dificuldade, inicialmente simplifica-se a abordagem para cada mecanismo de interação separadamente, para depois efetuar a análise de problemas mais complexos (FETTER, 1993; FREEZE e CHERRY, 1979). A seguir serão mostrados separadamente os principais mecanismos de transportes relacionados com as análises feitas neste artigo. Em seguida trata-se da formulação matemática para o fenômeno completo envolvendo os principais mecanismos envolvidos.

\subsection{Advecção}

Advecção é o mecanismo de transporte causado pela água em movimento, segundo vetores de fluxo. Com o deslocamento da água, os solutos presentes nela se deslocam na direção das linhas de fluxo com a velocidade igual à velocidade linear média da água (Equação 1), sem que ocorra alteração da concentração, e com taxa de transporte diretamente proporcional à velocidade da água (SHACKELFORD, 1993; FREEZE; CHERRY, 1979; FETTER, 1993). No caso unidimensional, a equação diferencial do transporte de solutos por advecção (FREEZE; CHERRY, 1979) é dada pela Equação 1.

$$
\frac{\partial C}{\partial t}=-v_{z} \frac{\partial C}{\partial z}
$$


onde $C$ representa a concentração do soluto $\left[\mathrm{M} / \mathrm{L}^{3}\right] ; v_{z}$ a velocidade da água nos poros na direção z [L/T]; to tempo [T]; e $z$ a coordenada vertical [L].

\subsection{Dispersão Hidrodinâmica}

O processo de difusão molecular não pode ser separado da dispersão mecânica no fluxo de água pelo solo. Esses dois mecanismos de transporte de solutos são combinados para que se obtenha o coeficiente de dispersão hidrodinâmica $D_{h}$. A dispersão hidrodinâmica pode ocorrer tanto na direção do fluxo, denominada de dispersão longitudinal, ou perpendicular ao fluxo, chamada então de dispersão transversal. Assim, os coeficientes de dispersão hidrodinâmica longitudinal $D_{l}\left[L^{2} / T\right]$ é (FETTER, 1993; FREEZE; CHERRY, 1979;BEAR, 1961):

$$
D_{l}=\alpha_{l} v_{z}+D^{*}
$$

onde $v_{z}$ é a velocidade da água nos poros na direção z [L/T]; $D^{*}$ é o coeficiente de difusão efetiva $\left(D^{*}=\tau D\right)$, e $\tau$, o fator de tortuosidade do solo [ ]; $\alpha_{l}$ é o coeficiente de dispersividade longitudinal [L]; e, $\alpha_{l} v_{z}$ é coeficiente de dispersão mecânica $\left[\mathrm{L}^{2} / T\right]$.

A dispersividade é provavelmente dependente da escala, com valores maiores para maiores distâncias de transporte (PICKENS; GRISAK, 1981). As dispersividades longitudinal e transversal são expressas respectivamente por:

$$
\alpha_{l}=\beta_{l} d
$$

em que $d$ corresponde ao diâmetro interno do poro [L]; $\beta_{l}$ tem valores da ordem de 1,75.

Desta forma, a Equação 3 pode ser reescrita (SCHEIDEGGER, 1963) como:

$$
D_{l}=1,75 d v_{z}+D^{*}
$$


De acordo com Fetter (1993) a dispersividade é aproximadamente 10\% do comprimento analisado.

\subsection{Fator de Retardamento}

Devido aos diversos mecanismos físicos, químicos e biológicos que ocorrem na interação entre a solução e o solo, a velocidade real de percolação da água, $v$, difere da velocidade do soluto, $v_{c}$, Equação 5.

$$
R_{d}=\frac{v}{v_{c}}=\frac{v t_{c}}{L}
$$

em que $t_{c}$ representa $o$ tempo necessário para o centro de massa do soluto migrando alcance o final da coluna de comprimento $L$.

O retardamento é um dos principais parâmetros a ser obtidos nos estudos do transporte de solutos nos meios porosos. Quando os solutos não interagem com o meio poroso (não reativos) o $R_{d}$ é 1 , representando que a velocidade de percolação do contaminante é a mesma que da água. Solutos com $R_{d}>1$ são chamados de solutos reativos (SHAKELFORD, 1994; FREEZE; CHERRY,1979). A Figura 1 ilustra a curva característica do transporte para situação com e sem retardamento.

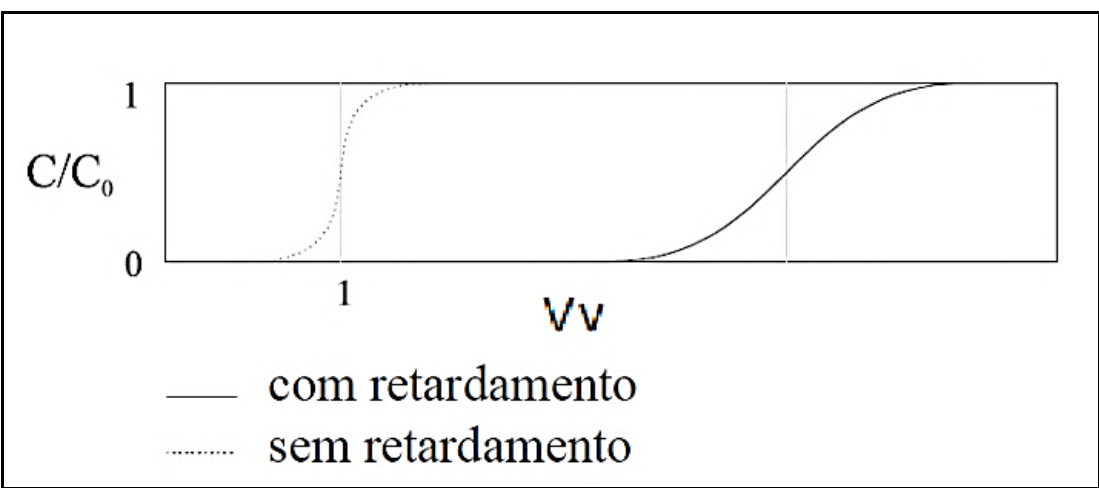

Figura 1 - Curva característica do transporte incluindo o efeito do retardamento (Modificado de FREEZE; CHERRY, 1979) 


\section{ABORDAGEM PROBABILÍSTICA DO TRANSPORTE DE CONTAMINANTE}

Sabe-se que os problemas de transporte de contaminantes possuem incertezas intrínsecas (ou inerentes) associadas, isto é, incertezas correspondentes à aleatoriedade natural dos fenômenos físicos, químicos, biológicos e atmosféricos aos quais possam estar relacionados. Estas incertezas se manifestam desde a heterogeneidade de base da formação do aquífero até a incerteza relativa às propriedades químicas, físicas e biológicas do contaminante a ser libertado e transportado (HAMED, BEDIENT e CONTE,1996; LI et al., 2005, BAALOUSHA e KÖNGETER, 2006).

A estimativa dessas incertezas por muito tempo foi realizada por meio de métodos puramente determinísticos. No entanto, inserindo as considerações acerca das incertezas físicas nos modelos determinísticos sobre um sistema, tem-se então um modelo probabilístico que permite uma avaliação mais adequada e racional do problema e, assim, a determinação da confiabilidade de um determinado sistema. A confiabilidade é a probabilidade de um evento favorável acontecer (sobrevivência). Por outro lado, a probabilidade de falha é a probabilidade de que um evento indesejado aconteça (falha). Assim, a confiabilidade e probabilidade de falha são complementares, ou seja:

$$
P(S)+P(F)=1
$$

onde $P$ é o operador probabilidade, $S$ é o evento sobrevivência e $F$ é o evento falha.

De maneira simplificada, a Equação (6) pode ser reescrita da seguinte maneira.

$$
P_{f}=1-P_{s}
$$

onde $P_{f}$ representa a probabilidade de falha e $P_{s}$, a sobrevivência o confiabilidade.

Agora, podemos definir à análise de confiabilidade como a estimativa do valor da probabilidade de falha ou da probabilidade de sobrevivência. Por simplicidade, neste estudo, o resultado da análise de confiabilidade é a probabilidade de falha. 
O transporte de contaminantes envolve parâmetros que são intrinsecamente variáveis e incertos. Assim, estes parâmetros podem ser tratados desde o ponto de vista da análise de confiabilidade como sendo variáveis aleatórias (VA). As VA que representam os parâmetros do problema são agrupados e representados pelo vetor $\mathbf{X}$. Desta maneira, o desempenho de um determinado comportamento é descrito através da sua função de performance, $g(\mathbf{X})$. Quando a função de performance adota um valor nulo, $g(\mathbf{X})=0$, divide o espaço amostral nos domínios de segurança e falha:

$$
\begin{aligned}
& \Omega_{s}=\{\mathbf{X} \mid g(\mathbf{X})>0\} \quad \text { domínio de segurança } \\
& \Omega_{f}=\{\mathbf{X} \mid g(\mathbf{X}) \leq 0\} \quad \text { domínio de falha }
\end{aligned}
$$

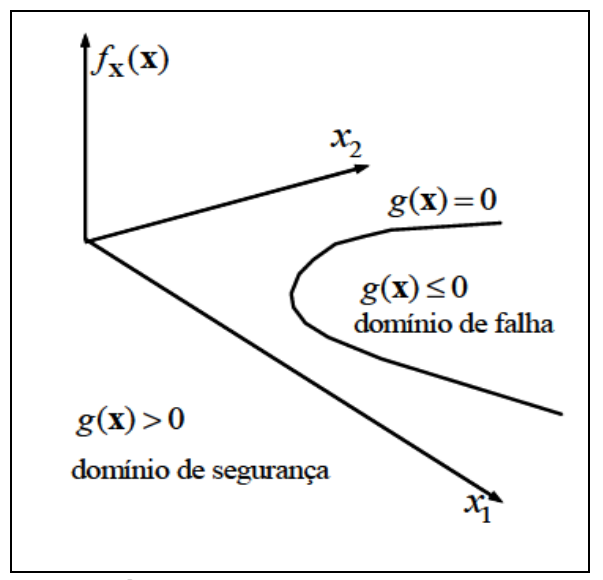

Figura 2 - Equação de estado limite e domínio de falha e de segurança (BECK, 2011)

Esta equação $g(\mathbf{X})=0$ é conhecida como a superfície de estado limite, LS. Assim, a probabilidade de falha, $P_{f}$, é a quantificação de probabilidade do vetor $\mathbf{X}$ adotar um valor dentro do domínio de falha, $\Omega_{f}$. Matematicamente, isto é equivalente a:

$$
P_{f}=\int_{\Omega_{f}} f_{\mathbf{X}}(x) \mathrm{d} x
$$

onde: $f_{\mathbf{X}}(x)$ é a função de densidade de probabilidade conjunta de $\mathbf{X}$. 


\subsection{Simulação de Monte Carlo}

Neste estudo, a abordagem do problema foi realizada através do uso da simulação de Monte Carlo. O método de confiabilidade por simulação de Monte Carlo (SMC) é um método considerado como "exato" desde que o número de realizações tenda ao infinito. Ou seja, que a precisão de valor depende da quantidade de amostras. Devido a que o número de amostras não pode ser infinito, existem técnicas para a estimativa dos erros de inferência (RUBINSTEIN, 2008; HONJO, 2008).

Este método consiste em fazer realizações das variáveis aleatórias através do sorteio do valor destas seguindo as suas próprias distribuições e correlações. Neste ponto, a geração de números aleatórios é fundamental. Esta geração pode ser realizada com funções para a geração de números pseudo-aleatórios (RUBINSTEIN, 2007). Alguns pacotes de software comerciais já tem gerados implementados internamente. Por outro lado, existem na internet geradores de números aleatórios reais. Contudo, nesta pesquisa foi utilizado o gerador automático do Mathematica 9.0 (WOLFRAM RESEARCH, 2013).

Uma vez feito o número total de realizações, a média do estimador da probabilidade de falha é calculado como o cociente entre o número de amostras que apresentaram falha e o número total de realizações (Equação (10)).

$$
\mathrm{E}\left[P_{f}\right]=P_{f}=\frac{n_{\text {falhas }}}{n_{\text {total }}}
$$

E a variância do estimador é:

$$
\operatorname{var}\left[P_{f}\right]=\frac{1}{n_{\text {total }}} P_{f}\left(1-P_{f}\right)
$$

Por outro lado, seguindo a critério de desigualdade de Chebyshev o número necessário de amostras que precisam ser realizadas para satisfazer um erro limite máximo $\varepsilon$ com um nível de confiança superior a $\alpha$ é dado por: 


$$
n_{\text {total }}>\frac{P_{f}\left(1-P_{f}\right)}{(1-\alpha) \varepsilon^{2}}
$$

\section{EXEMPLO DE APLICAÇÃO}

\subsection{Definição do modelo de transporte}

Para o exemplo analisado neste artigo foi adotado um modelo de transporte unidimensional considerando a presença de retardamento. Assim sendo, utilizou-se a Equação (13), para que se obtivesse a concentração no espaço e no tempo $C(x, t)$. No caso de problemas reais de contaminação, são necessárias análises bi ou tridimensionais. Para estes casos, métodos numéricos devem ser utilizados para que se obtenha a solução das equações de transporte (FREEZE e CHERRY, 1979), mas para o exemplo de aplicação unidimensional ela representa bem o transporte de contaminantes em meio poroso saturado.

$$
R_{d} \frac{\partial C}{\partial t}=D_{h x} \frac{\partial^{2} C}{\partial x^{2}}-v_{x} \frac{\partial C}{\partial x}
$$

em que $R_{d}$ é o fator de retardamento, onde $C$ representa a concentração do soluto [M/L $\left.{ }^{3}\right]$; $v_{x}$ a velocidade da água nos poros na direção $x[\mathrm{~L} / \mathrm{T}] ;$ e, $t$ o tempo [T]; $D_{h x} 0$ coeficiente de dispersão hidrodinâmica na direção $x\left[\mathrm{~L}^{2} / T\right]$.

Ogata (1958) e Ogata e Banks (1961) propuseram uma equação (Equação 14) que é solução para a equação diferencial parcial (Equação 13) que governa o processo de transporte advectivo-dispersivo em um meio homogêneo e isotrópico, com as condições de contorno adequadas e um fluxo constante através da coluna de solo.

$$
\frac{C}{C_{0}}=\frac{1}{2}\left[\operatorname{erfc}\left(\frac{L-v t}{2 \sqrt{D_{h} t}}\right)+\exp \left(\frac{v L}{D_{h}}\right) \operatorname{erfc}\left(\frac{L+v t}{2 \sqrt{D_{h} t}}\right)\right]
$$


em que $L$ o comprimento da fonte de contaminação [L] quando a concentração de saída é observada no final da coluna de solo, $v$ a velocidade linear média $\left[\mathrm{LT}^{-1}\right], t$ o tempo $[T] \mathrm{e}$ erfc é a função erro complementar.

Para o caso de solutos reativos, ou seja, que possuem um fator de retardamento $(R d)$, os parâmetros ve $D h$ da Equação 14 devem ser divididos pelo $R d$.

A erfc se relaciona com a distribuição normal o que significa que a solução descrita pela Equação 41 é normalmente distribuída, como esperado para processos de difusão. A erfc relaciona-se com a função erro, erf, da seguinte forma:

$$
\operatorname{erfc}(\beta)=1-\operatorname{erf}(\beta)
$$

O valor de erfc ( $\beta$ ) é 0 para qualquer valor positivo de $\beta$ maior do que 3,0 e 1,0 para $\beta$ igual a zero. Para valores negativos de $\beta$ a erfc é computada a partir das seguintes relações:

$$
\operatorname{erfc}(-\beta)=1+\operatorname{erf}(\beta)
$$

Sendo que a erf $\beta$ é definida como:

$$
\operatorname{erf}(\beta)=\frac{2}{\sqrt{\pi}} \int_{0}^{\beta} e^{-t^{2}} d t
$$

A Equação 17 não pode ser resolvida analiticamente, no entanto, os valores de $\beta$ são tabulados por diversos autores, entre eles Freeze e Cherry (1979). Além disso, ela pode ser aproximada pela expressão analítica:

$$
\operatorname{erf}(\beta)=\sqrt{1-\exp \left(\frac{-4 \beta^{2}}{\pi}\right)}
$$




\subsection{Definição das variáveis do problema}

Neste exemplo consideramos como variáveis aleatórias independentes a condutividade hidráulica, a porosidade e a dispersividade, assim como Hamed, Bedient e Conte (1996) e Hamed e Bedient (1999). As variáveis aleatórias dependentes são a velocidade linear média e o coeficiente de dispersão hidrodinâmica. A Tabela 1 mostra as distribuições estatísticas e seus parâmetros para as variáveis aleatórias

Tabela 1 Variáveis aleatórias: distribuições e seus parâmetros

\begin{tabular}{cccc}
\hline V.A independentes & Distribuição & Parâmetros & Autor \\
\hline $\mathbf{K}(\mathbf{m} / \mathbf{s})$ & $\mathrm{LN}$ & $\begin{array}{c}\text { Média }=5,0 \mathrm{E} 10-5 ; \\
\text { Desv. }=5,0 \mathrm{E} 10-5 \\
\text { Média }=5,0 ;\end{array}$ & \\
Alfa $(\mathbf{m})$ & $\mathrm{N}$ & Hamed, Bedient e \\
& $\mathrm{Desv}=1,5$ & Conte (1996) \\
$\mathbf{n}$ & $\mathrm{U}$ & Intervalo: $0,3-0,5$ & \\
& & & \\
\hline
\end{tabular}

As outras variáveis do problema foram assumidas como determinísticas, a saber: gradiente hidráulico, $i$, concentração inicial da solução contaminante, $C_{0}$, fator de retardamento, $R d$, comprimento da fonte de contaminação, $L$, tempo de análise, tanáise, e concentração objetivo, $\mathrm{C}_{\text {objetivo. }}$.

A concentração objetivo adotada neste exemplo foi de $5 \mathrm{mg} / \mathrm{m}^{3}$, conforme adotado por CETESB (2005) para Benzeno. No entanto, destaca-se que não se considerou o real comportamento do benzeno no solo, apenas utilizou-se sua $\mathrm{C}_{\text {objetivo }}$ a título de exemplo da aplicação da Simulação de Monte Carlo.

O comprimento da fonte de contaminação foi de 30 metros, gradiente hidráulico de 0,025 e o fator de retardamento utilizado foi de 7 . Variaram-se as concentrações iniciais de 0,5 a $1000 \mathrm{mg} / \mathrm{m}^{3}\left(0,5,5,10,15,25,50,100,500\right.$ e $\left.1000 \mathrm{mg} / \mathrm{m}^{3}\right)$. Todas as análise foram feitas para tanálise de 5 e 10 anos, conforme sugere CETESB (2007) como sendo tempos necessários para ser realizada modelagem da expansão da pluma de contaminação dissolvida. 


\subsection{Definição da Função de Performance}

Para o caso de transporte de contaminantes o evento de falha pode ser definido como a ocorrência de uma concentração observada, $C_{\text {observada }}$, acima de uma concentração limite, $C_{\text {objetivo }}$, para um tempo máximo pré-estabelecido, $t_{\text {análise }}$ (SITAR e CAWLFIELD, 1987; JANG, SITAR e KIUREGHIAN, 1994; HAMED, BEDIENT e CONTE,1996; LI et al., 2005, BAALOUSHA e KÖNGETER, 2006). Desta forma, a função de performance utilizada foi:

$$
g_{t c}(\mathbf{X})=C_{\text {objetivo }}-C_{\text {observada }}(\mathbf{X})
$$

O valor da $C_{\text {observada }}$ é dependente das VA do problema e, por tanto, é função do vetor $\mathbf{X}$ de VA.

Assim, a probabilidade de que a concentração observada exceda a concentração objetivo é dada por (HAMED, BEDIENT e CONTE,1996):

$$
P_{f}=P[g(\mathbf{X}) \leq 0]=P\left[C_{\text {objetivo }} \leq C_{\text {observada }}(\mathbf{X})\right]=\int_{g(\mathbf{X}) \leq 0} f_{\mathbf{X}}(x) \mathrm{d} x
$$

A máxima probabilidade de falha adotada neste artigo como aceitável é de $10 \mathrm{E}-5$, conforme propõe CETEBS (2006) por meio dos níveis aceitáveis baseados no risco para o Estado de São Paulo.

\section{Resultados e Discussões}

O processo de simulação empregou $10^{7}$ realizações de cada VA em cada concentração inicial $C_{0}$. Este processo foi realizado em uma planilha Mathematica 9.0. Com as simulações de Monte Carlo para $t_{\text {analise }}$ de 5 anos para os diversos valores de concentração inicial, contatou-se que ela variou de 0,5 a $25 \mathrm{mg} / \mathrm{m}^{3}$ a probabilidade de falha permaneceu dentro do limite de probabilidade aceitável especificado por CETESB (2006), i.e., abaixo de $10 \mathrm{E}-05$. No entanto, a partir de $50 \mathrm{mg} / \mathrm{m}^{3}$ nota-se o rápido 
incremento da probabilidade de falha, chegando até o extremo superior de $50 \%$ para uma concentração de $1000 \mathrm{mg} / \mathrm{m}^{3}$ (Figura 2).

As simulações de Monte Carlo para $t_{\text {analise }}$ de 10 anos, considerando novamente diversos valores de $C_{0}$, mostram que quando a mesma variou de 0,5 a $10 \mathrm{mg} / \mathrm{m}^{3}$ a probabilidade de falha permaneceu abaixo de 10E-05. No entanto, o aumento de 10 para $15 \mathrm{mg} / \mathrm{m}^{3}$ mostrou um incremento da probabilidade de falha em quatro ordens de grandeza, passando de $1.00 \mathrm{E}-07$ para $5.86 \mathrm{E}-03$. A partir de $25 \mathrm{mg} / \mathrm{m}^{3}$ constatam-se altas probabilidade de falha, variando de $23 \%$ para a concentração inicial de $25 \mathrm{mg} / \mathrm{m} 3$ até 98,5 e $99 \%$ para 500 e $1000 \mathrm{mg} / \mathrm{m} 3$, respectivamente (Figura 2).

Ao analisar os resultados em relação aos diferentes $t_{\text {analise }}$ nota-se que com o aumento do tempo ocorre aumento das probabilidades de falha para uma mesma concentração inicial. Isto porque o contaminante continua a se mover no perfil do solo e quanto maior o tempo em que ele permanecer no solo, maior será a probabilidade de que ele alcance a profundidade de interesse, mesmo com os efeitos de retardamento atuantes. Além disso, para o tempo de análise de 10 anos, constata-se um aumento mais rápido das probabilidades para a mesma variação da $\mathrm{C}_{0}$, quando comprado com $0 t_{\text {analise }}$ de 5 anos. A Figura 2 dois ilustra toso esses resultados. 


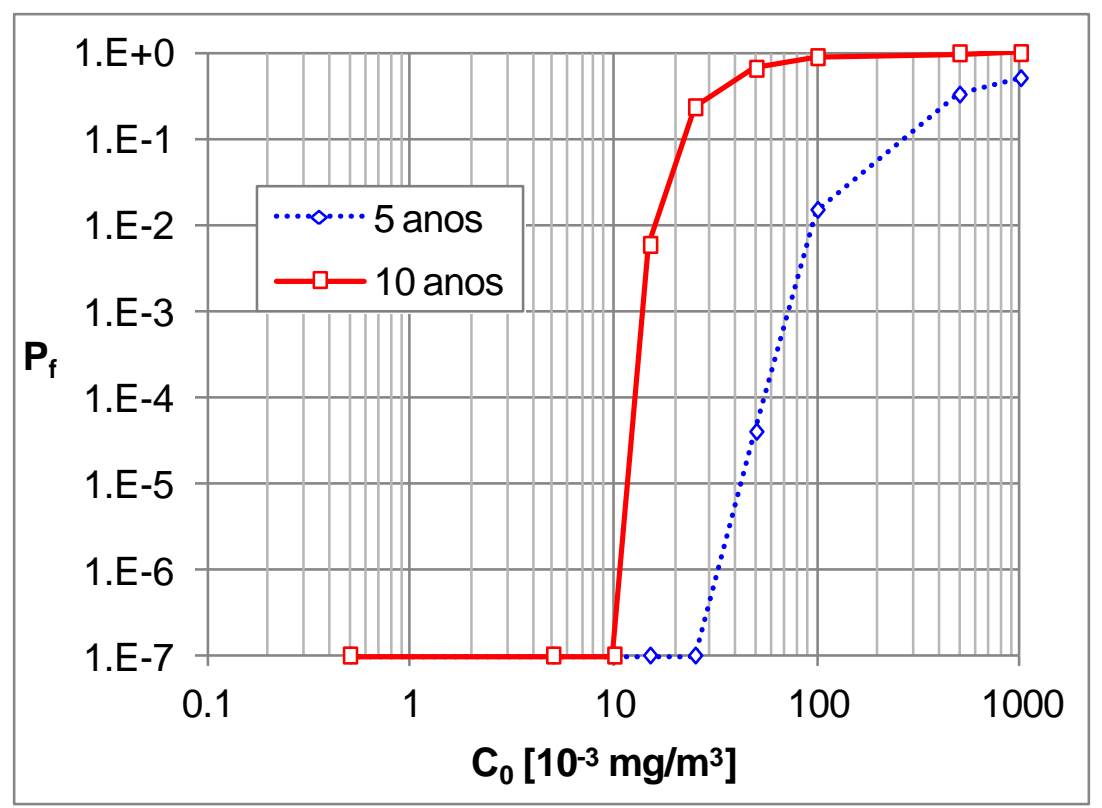

Figura 2 Variações da probabilidade falha com a concentração inicial e com o tempo de análise

Com isso, percebe-se que pequenas variações na concentração inicial podem ter efeitos muito sérios em termos de risco relacionado a determinado vazamento de contaminante no solo, já que o risco é diretamente dependente da probabilidade de falha, como exposto acima. Esses resultados chamam a atenção para a necessidade de rápida contenção e identificação de vazamentos, reduzindo o tempo bem como a concentração inicial da contaminação. Assim reduzir-se-ão as probabilidades de falha e consequentemente, os riscos envolvidos.

\section{CONSIDERAÇÕES FINAIS}

Com este trabalho pôde-se concluir que as análises probabilísticas são de extrema importância nos estudos de transporte de contaminantes dada as incertezas intrínsecas relacionadas. A análise das simulações de Monte Carlo permitiu que se notassem os efeitos de pequenas alterações na concentração inicial na probabilidade de falha, chegando a ocorrer um incremento de quatro ordens de grandeza na probabilidade de falha quando a concentração inicial variou de 10 para $15 \mathrm{mg} / \mathrm{m}^{3}$. A despeito disto, concluiu-se que com o aumento do tempo de análise ocorre aumento das probabilidades de falha para uma mesma concentração inicial. Por fim, este trabalho evidencia a 
necessidade de rápida contenção e identificação de vazamentos, reduzindo as probabilidades de falha e consequentemente, os riscos envolvidos.

\section{REFERÊNCIAS}

BAALOUSHA, H.; KÖNGETER, J. Stochastic modelling and risk analysis of groundwater pollution using FORM coupled with automatic differentiation. Advances in water resources, v. 29, n. 12, p. 1815-1832, 2006.

BEAR, J. Some experiments in dispersion. Journal of Geophysical Research, v. 66, $\mathrm{n}$. 8, p. 2455-2467, 1961.

Beck AT. Curso de confiabilidade estrutural.Versão 2011. São Carlos. 2011. 220p.

COMPANHIA AMBIENTAL DO ESTADO DE SÃO PAULO. Decisão de diretoria no 1952005-E, de 23 de novembro de 2005. Dispõe sobre a aprovação dos Valores Orientadores para Solos e Águas Subterrâneas no Estado de São Paulo - 2005, em substituição aos Valores Orientadores de 2001, e dá outras providências.

. Tabelas de referência níveis aceitáveis baseados no risco estado de São Paulo, sub anexo I do anexo vll. In: Ações corretivas baseadas em risco (ACBR) aplicadas a áreas contaminadas com hidrocarbonetos derivados de petróleo e outros combustíveis líquidos - procedimentos, anexo VII, 2006, 59p.

.Decisão de diretoria no 103-2007/C/E, de 22 de junho de 2007. Dispõe sobre Dispõe sobre o procedimento para gerenciamento de áreas contaminadas.

FETTER, C. W. Contaminant hydrogeology. Prentice Hall, Upper Saddle River, EUA, 1993. 500p.

FREEZE, R. A. et al. Hydrogeological decision analysis: 1. A framework. Ground Water, v. 28, n. 5, p. 738-766, 1990.

FREEZE, R. A.; CHERRY, J. A. Groundwater, Prentice-Hall, Englewood Cliffs, Nova Jersey 1979. $604 \mathrm{p}$.

HAMED, M. M.; BEDIENT, P. B. Reliability-based uncertainty analysis of groundwater contaminant transport and remediation. United States Environmental Protection Agency (USEPA). Report EPA/600/R-99/028. 1999, 82p. 
HAMED, M. M.; BEDIENT, P. B.; CONTE, J. P. Numerical stochastic analysis of groundwater contaminant transport and plume containment. Journal of Contaminant Hydrology, v. 24, n. 1, p. 1-24, 1996.

HIRATA, R.C.A. Os recursos hídricos subterrâneos e as novas exigências ambientais: aspectos teóricos para a definição de proteção e controle. Revista do Instituto Geológico. São Paulo. v. 1, n. 12, p. 39-62, 1993.

HONJO, Y. Monte Carlo simulation in reliability analysis. In: K.-K. Phoon (Editor), Reliability-Based Design in Geotechnical Engineering: Computations and Applications.2008, p. 169-191.

INTERNATIONAL SOCIETY OF SOIL MECHANICS AND GEOTECHNICAL ENGINEERING. TC304 - Glossary of Risk Assessment Terms - Version 1, July 2004. Technical Committee on Risk Assessment and Management. Online Version, 2004. 7 p.

JANG, Y.; SITAR, N.s; DER KIUREGHIAN, A. Reliability analysis of contaminant transport in saturated porous media. Water Resources Research, v. 30, n. 8, p. 2435-2448, 1994.

LEMMING, G.; FRIIS-HANSEN, P.; BJERG, P. L. Risk-based economic decision analysis of remediation options at a PCE-contaminated site. Journal of environmental management, v. 91, n. 5, p. 1169-1182, 2010.

LI, Y. et al. Stochastic modeling of the permeability of randomly generated porous media. Advances in water resources, v. 28, n. 8, p. 835-844, 2005.

Lu Q, Sun H-Y, Low BK. Reliability analysis of ground-support interaction in circular tunnels using the response surface method. Jounal of Rock Mechanics\& Minning Sciences; (48) (2011),pp. 1329-1343.

OGATA, A. Dispersion in porous media. 1958. 121 p. (Tese de Doutorado). Northwestern University, 1958.

OGATA, A., BANKS, R. B. A solution of the differential equation of longitudinal dispersion in porous media. US Geological Survey Artigo Profissional n. 411-A, USGS, Washington D.C. 1961.

PICKENS, J. F.; GRISAK, G. E. Scale-dependent dispersion in a stratified granular aquifer.Water Resources Research, v. 17, n. 4, p. 1191-1211, 1981.d region. Plant Soil and Environmental, v.52, n.8, p.335-344. 2006

RUBINSTEIN, R.; KROESE, D. Simulation and the Monte Carlo Method. 2nd. ed.: Wiley, 2008. ISBN 9780470177945. 
SCHEIDEGGER, A. E. Physics of flow through porous media. Toronto; University of Toronto, 1963. 313p.

SHACKELFORD, C D. Critical concepts for column testing. Journal of Geotechnical Engineering, v. 120, n. 10, p. 1804 -1828, 1994.

SITAR, N.; CAWLFIELD, J. D.; DER KIUREGHIAN, A. First-order reliability approach to stochastic analysis of subsurface flow and contaminant transport. Water Resources Research, v. 23, n. 5, p. 794-804, 1987.

WOLFRAM RESEARCH INC. Mathematica. Version 9.0. Champaign, Illinois. 2012. 